\title{
Pharmacological inhibition of poly(ADP-ribose) polymerase activity down-regulates the expression of syndecan-4 and Id-1 in endothelial cells
}

\author{
PEDRO MIGUEL LACAL ${ }^{1}$, LUCIO TENTORI ${ }^{2}$, ALESSIA MUZI ${ }^{2}$, FEDERICA RUFFINI ${ }^{1}$, \\ ANNALISA SUSANNA DORIO ${ }^{2}$, WEIZHENG XU ${ }^{3}$, DIEGO ARCELLI ${ }^{1}$, JIE ZHANG ${ }^{3}$ and GRAZIA GRAZIANI ${ }^{2}$ \\ ${ }^{1}$ Laboratory of Molecular Oncology, 'Istituto Dermopatico dell'Immacolata'-IRCCS, Via Monti di Creta 104; \\ 00167 Rome; ${ }^{2}$ Department of Neuroscience, University of Rome 'Tor Vergata', Via Montpellier 1, \\ 00133 Rome, Italy; ${ }^{3}$ Eisai, 6611 Tributary Street, Baltimore, MD 21224, USA
}

Received October 8, 2008; Accepted November 28, 2008

DOI: 10.3892/ijo_00000213

\begin{abstract}
Poly(ADP-ribose) polymerase (PARP) is a family of nuclear proteins which regulate a number of cell functions, such as DNA repair, transcription, remodelling of chromatin structure, cell division and cell death. We and others have recently demonstrated that down-regulation of cellular PARP activity, using pharmacological inhibitors, impairs a number of endothelial functions and angiogenesis. In the present study, we investigated the potential mechanisms underlying the antiangiogenic effect exerted by the potent PARP inhibitor GPI 15427 , analyzing gene expression in human endothelial cells shortly after treatment with this compound. Analysis of gene and protein expression indicated that a 2-h exposure of human endothelial cells to GPI 15427 induced a rapid decrease of syndecan-4 (SDC-4), a transmembrane protein involved in modulation of cell signalling during angiogenesis that plays a role in endothelial cell migration and adhesion. Moreover, treatment with the PARP inhibitor induced a reduction of a helix-loop-helix transcription factor, the inhibitor of DNA binding-1 (Id-1), also implicated in the control of endothelial functions. We suggest that the inhibitory effect exerted by GPI 15427 on the angiogenic process is likely due to the reduced activity of specific transcription factors, such as Oct-1 and CREB that contribute to the regulation of SDC-4 and Id-1 expression, respectively. In conclusion, these results strongly suggest that PARP activity is capable of modulating molecules required for endothelial cell migration, adhesion, proliferation or differentiation during the angiogenic process.
\end{abstract}

Correspondence to: Dr Grazia Graziani, Department of Neuroscience, University of Rome 'Tor Vergata', Via Montpellier 1, 00133 Rome, Italy

E-mail: graziani@uniroma2.it

Key words: poly(ADP-ribose) polymerase, angiogenesis, endothelial cells

\section{Introduction}

Poly(ADP-ribose) polymerase (PARP) is a family of eukaryotic nuclear proteins which plays a key role in regulating DNA repair, transcription, chromatin function, cell division and cell death (1). PARPs synthesize polymers of ADP-ribose using $\mathrm{NAD}^{+}$as a substrate and catalyse the attachment of poly(ADP-ribose) to acceptor proteins modifying their function. This post-translational modification is transient due to the prompt degradation of the polymers by poly(ADPribose) glycohydrolase. Among the PARP family members, PARP-1 is responsible for most of the cellular poly(ADPribosyl)ating activity and its primary targets include PARP-1 itself, histones and a variety of transcription factors. So far, only PARP-1 and PARP-2 are known to be activated by DNA damage, acting as sensor of genotoxic damage and co-ordinating repair (1). However, PARP-2 is less active than PARP-1 in the response to DNA damage and unique PARP-2 functions in various differentiation processes have been recently identified (2).

PARP-1 and -2 are involved in the base excision repair (3), which corrects DNA base lesions including N-methylpurines induced by methylating agents and oxidized bases or single strand breaks generated by ionizing radiations (4). Moreover, PARP-1 modulates the repair of double-strand breaks through its interaction with components of the non-homologous end joining (5). In its poly(ADP-ribos)ylated form PARP-1 counteracts the action of topoisomerase I poisons facilitating resealing of DNA strand (6). Recently, it has been demonstrated that PARP-1 may play a role also in the nucleotide excision repair pathway (7). Thus, PARP inhibitors have been exploited in cancer treatment to increase the efficacy of chemotherapeutic agents such as temozolomide, dacarbazine, carboplatin, irinotecan, topotecan or of radiotherapy, and are currently under evaluation in phase I and II clinical trials for the treatment of refractory solid and hematological tumors (4, www.clinicaltrials.gov). PARP inhibitors are also investigated as single agents for the therapy of ovarian or breast cancer carrying mutations of breast cancer associated gene 1 and 2 (BRCA-1 and BRCA-2) which are involved in the homologous 
recombination repair machinery $(8,9$, www.clinicaltrials gov).

In addition to DNA repair mechanisms, targeting PARP-1 has been shown to suppress angiogenesis, which is an essential requirement for the growth of tumours. We recently demonstrated a direct involvement of PARP-1 function in angiogenesis as indicated by the reduction of blood vessel neo-formation in response to angiogenic stimuli observed in PARP-1 KO mice (10). Similar results were obtained in animals treated with the potent PARP inhibitor GPI 15427 (10). The observed anti-angiogenic effect of GPI 15427 is associated with a decrease of endothelial cell migration in response to angiogenic factors, such as the vascular endothelial growth factor (VEGF) or the placenta growth factor, and cannot be directly related to increased DNA damage, since it is observed at drug concentrations that do not affect the viability and the proliferative potential of endothelial cells. Similar anti-angiogenic effects were obtained also with other PARP inhibitors (11-13). Notably, abrogation of PARP-1 expression by stable gene silencing reduced the aggressiveness of melanoma and this effect was associated with a decreased vasculature formation within the tumour (14). Altogether, these data strongly suggest that PARP inhibitors may also exert antitumor activity through a reduction of tumour-associated neo-angiogenesis, independently on their effect on DNA repair.

In the present study, we demonstrate that a short exposure of human endothelial cells to the PARP inhibitor GPI 15427 induces a rapid decrease of the expression of syndecan-4 (SDC-4), a transmembrane protein involved in modulating cell signalling during angiogenesis, and a later reduction of the expression of inhibitor of DNA binding-1 (Id-1), a helixloop-helix transcription factor which regulates endothelial functions.

\section{Materials and methods}

Cell culture and treatment with PARP inhibitor. The immortalized human endothelial cell line HUV-ST was generated as previously described (15). Cells were maintained in culture in endothelial growth factor medium (EGM-2; Clonetics, BioWhittaker Inc., Walkersville, MD, USA) supplemented with $0.4 \mathrm{mg} / \mathrm{ml}$ geneticin and $5 \mu \mathrm{g} / \mathrm{ml}$ puromycin.

For PARP inhibition in endothelial cells, the recently developed compound GPI 15427 [10-(4-methyl-piperazin-1ylmethyl)-2H-7-oxa-1,2-diaza-benzo[de]anthracen-3-one, Eisai, Baltimore, MD, USA] was used (16). The PARP inhibitor stock solution (1 $\mathrm{mM})$ was prepared by dissolving GPI 15427 in $70 \mathrm{mM}$ PBS without potassium.

PARP activity assay. To measure total cellular PARP activity, HUV-ST cells $\left(5 \times 10^{6}\right)$ were lysed in $0.5 \mathrm{ml}$ of a buffer containing $0.1 \%$ Triton $\mathrm{X}, 50 \mathrm{mM}$ Tris- $\mathrm{HCl} \mathrm{pH} 8.0,0.6 \mathrm{mM}$ EDTA, $14 \mathrm{mM}$ B-mercaptoethanol, $10 \mathrm{mM} \mathrm{MgCl}_{2}$ and protease inhibitors. Proteins $(25 \mu \mathrm{g})$ were incubated with $2 \mu \mathrm{Ci}$ ${ }^{32} \mathrm{P}-\mathrm{NAD}^{+}$(GE Healthcare, Milan, Italy), $10 \mu \mathrm{M} \mathrm{NAD}^{+}, 50 \mathrm{mM}$

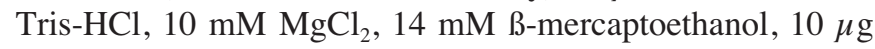
nuclease-treated salmon testes DNA. PARP activity was expressed as fmol of ${ }^{32} \mathrm{P}-\mathrm{NAD}^{+} / \mu \mathrm{g}$ of protein (16). For the analysis of the influence of GPI 15427 on cellular PARP, intact HUV-ST cells $\left(5 \times 10^{5}\right)$ were treated with the inhibitor and permeabilized with digitonin $(0.1 \mathrm{mg} / \mathrm{ml})$ in the presence of $0.25 \mu \mathrm{Ci}{ }^{3} \mathrm{H}_{-N A D}{ }^{+}$(PerkinElmer, Milan, Italy) (17).

\section{Microarray analysis of gene expression}

Gene expression profiling. Total cellular RNA was isolated from endothelial cells using RNeasy kits (Qiagen, Germantown, MD, USA). Preparation of labeled cRNA and hybridization (GeneChip Human Genome U133A array, Affymetrix Inc., High Wycombe, UK) was performed according to the Affymetrix GeneChip expression analysis manual.

Data analysis. Affymetrix GeneChip scanning was analyzed by a customized R language-based script (see www.r-project.org) which utilizes the Bioconductor packages (see www.bioconductor.org) for quality control analysis, data normalization, hierarchical cluster and identification of differentially expressed transcripts. Specifically, the 'gcrma package' was used for chip normalization and background correction; the 'vsn package' provided calibration and transformation of the probe intensities. The genefilter package was used to separate genes with high variance according to the interquartile range method.

Prediction analysis. Prediction analysis of microarrays (PAM) is a statistical technique for class prediction utilizing gene expression data using shrunken centroids. The method of nearest shrunken centroids identifies subsets of genes that best characterize each class (18). In order to test the internal consistency and to explore the relationship among untreated or GPI 15427-treated cell samples and underlying features of gene expression the unsupervised cluster analysis was followed by PAM R supervised class prediction package.

Differentially expressed genes. To identify genes differentially expressed in GPI 15427-treated and untreated cell samples, we used a significant analysis of microarrays (SAM) test, which uses permutation to identify significant differences between different groups. Analysis was performed using the $\mathrm{R}$ package 'samr' which was applied in order to adjust P-values using a false discovery rate to identify genes that were significantly different between groups (19).

The lists of genes modulated by treatment with GPI 15427 that are shown in the Results section correspond to the genes that resulted to be up- or down-modulated in both PAM and SAM analysis.

Gene function analysis. Regulated biological processes and molecular functions were identified by the GEPAS web-based application according to the Gene Ontology (http://www. geneontology.org) Consortium classification (20).

Western blot analysis. Time-course experiments of specific protein expression were performed in semi-confluent cultures of HUV-ST cells, growing in 6-well plates. Cells were treated with $2 \mu \mathrm{M}$ GPI 15427 for different periods of time; wells were, then, washed with PBS and cell lysates were prepared using a lysis buffer (150 mM NaCl, $1 \mathrm{mM}$ EDTA, $0.5 \% \mathrm{NP}-40,50 \mathrm{mM}$ Tris-HCl pH 8.0) containing phosphatase (PhoSTOP, Roche Diagnostics, Mannheim, Germany) and protease (Complete Mini, EDTA-free, Roche) inhibitors. Samples were run in a $10 \%$ SDS-polyacrylamide gel and proteins were then transferred to supported nitrocellulose membranes (Hybond-C; GE Healthcare). Membranes were blocked in blocking solution 
Table I. Up-modulated genes after GPI 15427 treatment of endothelial cells.

\begin{tabular}{|c|c|c|c|c|}
\hline Probe & Symbol & Description $^{\mathrm{a}}$ & Score $^{b}$ & Fold change $e^{b}$ \\
\hline 203035_s_at & PIAS3 & Protein inhibitor of activated STAT, 3 & 3.21 & 1.85 \\
\hline 220748_s_at & ZNF580 & Zinc finger protein 580 & 3.03 & 1.81 \\
\hline 209140_x_at & HLA-B & Major histocompatibility complex, class I, B & 2.41 & 1.60 \\
\hline 214459_x_at & HLA-C & Major histocompatibility complex, class I, C & 2.40 & 1.76 \\
\hline 200696_s_at & GSN & Gelsolin (amyloidosis, Finnish type) & 2.23 & 1.51 \\
\hline 201626_at & INSIG1 & Insulin-induced gene 1 & 2.22 & 1.83 \\
\hline 204067_at & SUOX & Sulfite oxidase & 2.21 & 1.62 \\
\hline 202245_at & LSS & Lanosterol synthase (2,3-oxidosqualene-lanosterol cyclase) & 2.21 & 1.85 \\
\hline 37462_i_at & SF3A2 & Splicing factor $3 \mathrm{a}$, subunit $2,66 \mathrm{kDa}$ & 2.21 & 1.63 \\
\hline 214246_x_at & MINK1 & Misshapen-like kinase 1 (zebrafish) & 2.17 & 1.59 \\
\hline
\end{tabular}

${ }^{a}$ The list corresponds to the genes that resulted to be modulated in both PAM and SAM analysis. ${ }^{\mathrm{b} S c o r e}$ and fold change parameters were obtained by SAM analysis.

(4\% BSA, 0.1\% Tween-20, 0.9\% NaCl, $20 \mathrm{mM}$ Tris- $\mathrm{HCl}$, $\mathrm{pH}$ 7.4) and incubated in the same solution overnight at $4{ }^{\circ} \mathrm{C}$ with primary antibodies.

The rabbit polyclonal antibodies anti-Id-1 (C20, diluted 1:200), anti-ATF-3 (C-19, diluted 1:200), anti-Oct-1 (C21, diluted 1:200) and anti- $\beta$-tubulin (H-235, diluted 1:1000), as well as the monoclonal antibodies anti-SDC-4 (5G9, diluted 1:200), anti-ATF-1/CREB (25C10G, diluted 1:200) and antiphosphotyrosine (PY20, diluted 1:200) were all from Santa Cruz Biotechnology (Santa Cruz, CA, USA); the monoclonal antibody anti-phospho-CREB/ATF-1 (1B6, diluted 1:1000) was purchased from Cell Signaling (Beverly, MA, USA). After washing with TBST buffer $(0.1 \%$ Tween-20, $0.9 \% \mathrm{NaCl}$, $20 \mathrm{mM}$ Tris-HCl, pH 7.4), membranes were incubated for $1 \mathrm{~h}$ at room temperature with the appropriate horseradishperoxidase conjugated secondary antibody (anti-rabbit or antimouse Ig from GE Healthcare, diluted 1:5000 in blocking solution). Membranes were then washed with TBST buffer and detection was carried out using the ECL Western blot detection reagents and Hyperfilm, both from GE Healthcare. Densitometric analysis was performed using a GS710 densitometer from Bio-Rad Laboratories (Hercules, CA, USA).

$N F-\kappa B$ and Oct-1 activation assays. Time-course experiments of transcription factors activity were performed in semiconfluent cultures of HUV-ST cells, growing in 6-well plates. Cells were treated with $2 \mu \mathrm{M}$ GPI 15427 for different periods of time and then lysed to prepare nuclear extracts. Nuclear proteins were prepared using the Active motif nuclear extract kit (Active Motif Europe, Rixensart, Belgium) according to the manufacturer's instructions. Activation of the $\mathrm{NF}-\kappa \mathrm{B}$ p65 subunit in $10 \mu \mathrm{g}$ of HUV-ST nuclear extracts was determined using an NF-кB p65 Enzyme-linked immunosorbent assay (ELISA)-based transcription factor assay kit (TransAM assay) (Active Motif Europe), according to the manufacturer's protocol. This kit contains a 96-well plate with immobilized oligonucleotides encoding an NF-кB consensus site (5'-GGGACTTTCC-3'). The active form of this transcription factor specifically binds to this oligonucleotide. The NF- $\mathrm{BB}$ detecting antibody recognizes an epitope on $\mathrm{p} 65$ that is accessible only when this polypeptide is activated and bound to its target DNA. A horseradish peroxidase-conjugated secondary antibody provides a sensitive colorimetric readout that is quantified by a Bio-Rad microplate reader 680 at $450 \mathrm{~nm}$ with a reference wavelength of $655 \mathrm{~nm}$. The positive control Jurkat nuclear extract, provided within the kit, was used to assess assay specificity.

Oct- 1 activity was assayed using a modified Oct-4 TransAM kit from Active Motif Europe. In brief, the 96-well plate with immobilized oligonucleotides encoding the octamer binding transcription factor family consensus sequence (5'-ATGCAAAT-3') was utilized to capture the activated Oct-1 protein present in the HUV-ST nuclear extracts $(10 \mu \mathrm{g})$. Then, the assay was performed according to the manufacturer's instructions using an anti-Oct-1 rabbit polyclonal antibody from Santa Cruz (C-21) diluted 1:1000. A nuclear extract from P19 cells, provided with the kit, was used as positive control

\section{Results}

Analysis of differential gene expression in untreated or GPI 15427-treated HUV-ST cells. HUV-ST cells were initially analyzed for PARP activity, measured in cell extracts in the presence of nuclease-treated salmon testes DNA and ${ }^{32} \mathrm{P}$ $\mathrm{NAD}^{+}$. The results indicated that total PARP activity of HUV-ST cells was $853 \pm 178 \mathrm{fmol} / \mu \mathrm{g}$ of protein. We then tested the ability of GPI 15427 to inhibit PARP activity of intact endothelial cells by exposure of HUV-ST cells for $1 \mathrm{~h}$ to graded concentrations of GPI $15427(0.1-2 \mu \mathrm{M})$, followed by permeabilization with digitonin in the presence of ${ }^{3} \mathrm{H}-\mathrm{NAD}^{+}$. The results indicated that GPI 15427 easily penetrated into the cells and inhibited PARP activity with an $\mathrm{IC}_{50}$ of $237 \pm 27 \mathrm{nM}$.

To investigate the early molecular changes after exposure of endothelial cells with GPI 15427, HUV-ST cells were treated for $2 \mathrm{~h}$ with $2 \mu \mathrm{M}$ GPI 15427 , a concentration that completely inhibits PARP activity and angiogenesis and that does not affect the growth and clonogenic survival of endothelial cells (10). Total RNA was extracted from untreated $(n=2)$ or drug-treated cultures $(n=2)$ and gene expression was evaluated by microarray analysis. The identification of the differentially expressed genes showed that most of these genes were down-regulated by drug treatment (Tables I and II). The genes demonstrating early modulated expression mainly 
Table II. Down-modulated genes after GPI 15427 treatment of endothelial cells.

\begin{tabular}{|c|c|c|c|c|}
\hline Probe & Symbol & Description $^{\mathrm{a}}$ & Score ${ }^{b}$ & $\begin{array}{c}\text { Fold } \\
\text { change }\end{array}$ \\
\hline 202071_at & SDC-4 & Syndecan 4 (amphiglycan, ryudocan) & -4.56 & 0.41 \\
\hline 210762_s_at & DLC1 & Deleted in liver cancer 1 & -4.44 & 0.31 \\
\hline 208937_s_at & Id-1 & Inhibitor of DNA binding 1, dominant negative helix-loop-helix protein & -4.34 & 0.22 \\
\hline 218237_s_at & SLC38A1 & Solute carrier family 38, member 1 & -4.14 & 0.38 \\
\hline 205321_at & EIF2S3 & Eukaryotic translation initiation factor 2 , subunit $3 \gamma, 52 \mathrm{kDa}$ & -3.77 & 0.32 \\
\hline 212582_at & OSBPL8 & Oxysterol binding protein-like 8 & -3.64 & 0.33 \\
\hline 201123_s_at & EIF5A & Eukaryotic translation initiation factor $5 \mathrm{~A}$ & -3.62 & 0.28 \\
\hline 211136_s_at & CLPTM1 & Cleft lip and palate associated transmembrane protein 1 & -3.58 & 0.43 \\
\hline 221806_s_at & SETD5 & SET domain containing 5 & -3.57 & 0.34 \\
\hline 200852_x_at & GNB2 & Guanine nucleotide binding protein ( $\mathrm{G}$ protein), $\beta$ polypeptide 2 & -3.47 & 0.41 \\
\hline 205924_at & RAB3B & RAB3B, member RAS oncogene family & -3.45 & 0.50 \\
\hline 213757_at & EIF5A & Eukaryotic translation initiation factor $5 \mathrm{~A}$ & -3.29 & 0.33 \\
\hline 209832_s_at & CDT1 & Chromatin licensing and DNA replication factor 1 & -3.28 & 0.42 \\
\hline 208777_s_at & PSMD11 & Proteasome (prosome, macropain) 26S subunit, non-ATPase, 11 & -3.24 & 0.46 \\
\hline 206445_s_at & PRMT1 & Protein arginine methyltransferase 1 & -3.20 & 0.49 \\
\hline 202416_at & DNAJC7 & DnaJ (Hsp40) homolog, subfamily C, member 7 & -3.19 & 0.54 \\
\hline 217756_x_at & SERF2 & Small EDRK-rich factor 2 & -3.17 & 0.40 \\
\hline 222244_s_at & TUG1 & RNA taurine upregulated gene 1 & -3.16 & 0.33 \\
\hline 204004_at & PAWR & PRKC, apoptosis, WT1, regulator & -3.03 & 0.29 \\
\hline 203109_at & UBE2M & Ubiquitin-conjugating enzyme E2M (UBC12 homolog, yeast) & -3.01 & 0.29 \\
\hline 210092_at & MAGOH & Mago-nashi homolog, proliferation-associated (Drosophila) & -3.00 & 0.43 \\
\hline 212015_x_at & PTBP1 & Polypyrimidine tract binding protein 1 & -3.00 & 0.54 \\
\hline 202976_s_at & RHOBTB3 & Rho-related BTB domain containing 3 & -2.98 & 0.50 \\
\hline 208750_s_at & ARF1 & ADP-ribosylation factor 1 & -2.93 & 0.42 \\
\hline 213980_s_at & CTBP1 & C-terminal binding protein 1 & -2.91 & 0.56 \\
\hline 201801_s_at & SLC29A1 & Solute carrier family 29 (nucleoside transporters), member 1 & -2.89 & 0.42 \\
\hline 211671_s_at & NR3C1 & Nuclear receptor subfamily 3 , group $\mathrm{C}$, member 1 (glucocorticoid receptor) & -2.88 & 0.54 \\
\hline 202407_s_at & PRPF31 & PRP31 pre-mRNA processing factor 31 homolog (S. cerevisiae) & -2.84 & 0.55 \\
\hline 203917_at & CXADR & Coxsackie virus and adenovirus receptor & -2.81 & 0.35 \\
\hline 203093_s_at & TIMM44 & Translocase of inner mitochondrial membrane 44 homolog (yeast) & -2.80 & 0.46 \\
\hline 222029_x_at & PFDN6 & Prefoldin subunit 6 & -2.71 & 0.57 \\
\hline 209042_s_at & UBE2G2 & Ubiquitin-conjugating enzyme E2G 2 (UBC7 homolog, yeast) & -2.70 & 0.46 \\
\hline 209208_at & MPDU1 & Mannose-P-dolichol utilization defect 1 & -2.69 & 0.57 \\
\hline 209264_s_at & TSPAN4 & Tetraspanin 4 & -2.68 & 0.47 \\
\hline 201164_s_at & PUM1 & Pumilio homolog 1 (Drosophila) & -2.62 & 0.49 \\
\hline 214383_x_at & KLHDC3 & Kelch domain containing 3 & -2.62 & 0.53 \\
\hline 202656_s_at & SERTAD2 & SERTA domain containing 2 & -2.61 & 0.46 \\
\hline 202839_s_at & NDUFB7 & NADH dehydrogenase (ubiquinone) 1 beta subcomplex, 7, $18 \mathrm{kDa}$ & -2.61 & 0.48 \\
\hline 213887_s_at & POLR2E & Polymerase (RNA) II (DNA directed) polypeptide E, $25 \mathrm{kDa}$ & -2.59 & 0.43 \\
\hline 205748_s_at & RNF126 & Ring finger protein 126 & -2.57 & 0.57 \\
\hline 212961_x_at & CXorf40B & Chromosome $\mathrm{X}$ open reading frame 40B & -2.57 & 0.47 \\
\hline 207088_s_at & SLC25A11 & Solute carrier family 25 (mitochondrial carrier; oxoglutarate carrier), member 11 & -2.56 & 0.55 \\
\hline 214911_s_at & BRD2 & Bromodomain containing 2 & -2.55 & 0.56 \\
\hline 208645_s_at & RPS14 & Ribosomal protein $\mathrm{S} 14$ & -2.54 & 0.57 \\
\hline 212125_at & RANGAP1 & Ran GTPase activating protein 1 & -2.53 & 0.45 \\
\hline 200968_s_at & PPIB & Peptidylprolyl isomerase B (cyclophilin B) & -2.50 & 0.46 \\
\hline 204212_at & ACOT8 & Acyl-CoA thioesterase 8 & -2.50 & 0.41 \\
\hline 212040_at & TGOLN2 & Trans-golgi network protein 2 & -2.50 & 0.44 \\
\hline 212251_at & MTDH & Metadherin & -2.49 & 0.42 \\
\hline 218206_x_at & SCAND1 & SCAN domain containing 1 & -2.47 & 0.56 \\
\hline 201865_x_at & $\mathrm{NR} 3 \mathrm{C} 1$ & Nuclear receptor subfamily 3 , group C, member 1 (glucocorticoid receptor) & -2.46 & 0.53 \\
\hline 204031_s_at & PCBP2 & Poly $(\mathrm{rC})$ binding protein 2 & -2.46 & 0.46 \\
\hline 218368_s_at & TNFRSF12A & Tumor necrosis factor receptor superfamily, member $12 \mathrm{~A}$ & -2.45 & 0.43 \\
\hline 217716_s_at & SEC61A1 & Sec61 alpha 1 subunit ( . cerevisiae) & -2.45 & 0.55 \\
\hline 204640_s_at & SPOP & Speckle-type POZ protein & -2.44 & 0.39 \\
\hline 208625_s_at & EIF4G1 & Eukaryotic translation initiation factor $4 \gamma, 1$ & -2.41 & 0.43 \\
\hline 201506_at & TGFBI & Transforming growth factor, $\beta$-induced, $68 \mathrm{kDa}$ & -2.38 & 0.59 \\
\hline 221516_s_at & SMCR7L & Smith-Magenis syndrome chromosome region, candidate 7-like & -2.38 & 0.60 \\
\hline
\end{tabular}


Table II. Continued

\begin{tabular}{|c|c|c|c|c|}
\hline Probe & Symbol & Description $^{\mathrm{a}}$ & Score $^{b}$ & $\begin{array}{c}\text { Fold } \\
\text { change }^{b}\end{array}$ \\
\hline 212863_x_at & СТВP1 & C-terminal binding protein 1 & -2.37 & 0.50 \\
\hline 200967_at & PPIB & Peptidylprolyl isomerase B (cyclophilin B) & -2.37 & 0.53 \\
\hline 212596_s_at & HMG2L1 & High-mobility group protein 2 -like 1 & -2.36 & 0.46 \\
\hline 201050_at & PLD3 & Phospholipase D family, member 3 & -2.34 & 0.48 \\
\hline 201075_s_at & SMARCC1 & $\begin{array}{l}\text { SWI/SNF related, matrix associated, actin dependent regulator of chromatin, } \\
\text { Subfamily c, member } 1\end{array}$ & -2.34 & 0.58 \\
\hline 218028_at & ELOVL1 & Elongation of very long chain fatty acids (FEN1/Elo2, SUR4/Elo3, yeast)-like 1 & -2.31 & 0.42 \\
\hline 207826_s_at & ID3 & Inhibitor of DNA binding 3, dominant negative helix-loop-helix protein & -2.31 & 0.45 \\
\hline 210183_x_at & PNN & Pinin, desmosome associated protein & -2.30 & 0.44 \\
\hline 212277_at & MTMR4 & Myotubularin related protein 4 & -2.30 & 0.58 \\
\hline 201040_at & GNAI2 & $\begin{array}{l}\text { Guanine nucleotide binding protein (G protein), } \alpha \text { inhibiting } \\
\text { Activity polypeptide } 2\end{array}$ & -2.28 & 0.56 \\
\hline 213236_at & SASH1 & SAM and SH3 domain containing 1 & -2.28 & 0.51 \\
\hline 208887_at & EIF3S4 & Eukaryotic translation initiation factor 3 , subunit $4 \delta, 44 \mathrm{kDa}$ & -2.28 & 0.59 \\
\hline 201095_at & DAP & Death-associated protein & -2.26 & 0.58 \\
\hline 213746_s_at & FLNA & Filamin A, $\alpha$ (actin binding protein 280 ) & -2.23 & 0.56 \\
\hline 204405_x_at & DIMT1L & DIM1 dimethyladenosine transferase 1-like (S. cerevisiae) & -2.23 & 0.61 \\
\hline 201862_s_at & LRRFIP1 & Leucine rich repeat (in FLII) interacting protein 1 & -2.22 & 0.50 \\
\hline 202646_s_at & CSDE1 & Cold shock domain containing E1, RNA-binding & -2.20 & 0.62 \\
\hline 218302_at & PSENEN & Presenilin enhancer 2 homolog (C.elegans) & -2.19 & 0.55 \\
\hline 209953_s_at & CDC37 & CDC37 cell division cycle 37 homolog ( . cerevisiae) & -2.19 & 0.50 \\
\hline 202223_at & STT3A & $\begin{array}{l}\text { STT3, subunit of the oligosaccharyltransferase complex, homolog A } \\
\text { (S. cerevisiae) }\end{array}$ & -2.18 & 0.55 \\
\hline 208685_x_at & BRD2 & Bromodomain containing 2 & -2.18 & 0.62 \\
\hline 209008_x_at & KRT8 & Keratin 8 & -2.18 & 0.51 \\
\hline 217802_s_at & NUCKS1 & Nuclear casein kinase and cyclin-dependent kinase substrate 1 & -2.17 & 0.57 \\
\hline 220272_at & BNC2 & Basonuclin 2 & -2.16 & 0.47 \\
\hline 214656_x_at & MYO1C & Myosin IC & -2.15 & 0.57 \\
\hline 202024_at & ASNA1 & ArsA arsenite transporter, ATP-binding, homolog 1 (bacterial) & -2.15 & 0.52 \\
\hline 212515_s_at & DDX3X & DEAD (Asp-Glu-Ala-Asp) box polypeptide 3, X-linked & -2.12 & 0.57 \\
\hline 202224_at & CRK & V-crk sarcoma virus CT10 oncogene homolog (avian) & -2.12 & 0.60 \\
\hline 218473_s_at & GLT25D1 & Glycosyltransferase 25 domain containing 1 & -2.11 & 0.54 \\
\hline 208447_s_at & PRPS1 & Phosphoribosyl pyrophosphate synthetase 1 & -2.11 & 0.56 \\
\hline 221744_at & WDR68 & WD repeat domain 68 & -2.10 & 0.51 \\
\hline 211177_s_at & TXNRD2 & Thioredoxin reductase 2 & -2.10 & 0.57 \\
\hline 212783_at & RBBP6 & Retinoblastoma binding protein 6 & -2.10 & 0.59 \\
\hline 200612_s_at & AP2B1 & Adaptor-related protein complex 2, 31 subunit & -2.09 & 0.54 \\
\hline 208978_at & CRIP2 & Cysteine-rich protein 2 & -2.07 & 0.65 \\
\hline 204980_at & CLOCK & Clock homolog (mouse) & -2.07 & 0.66 \\
\hline 200641_s_at & YWHAZ & $\begin{array}{l}\text { Tyrosine } 3 \text {-monooxygenase/tryptophan } 5 \text {-monooxygenase activation protein, } \\
\varsigma \text { polypeptide }\end{array}$ & -2.06 & 0.54 \\
\hline 201609_x_at & ICMT & Isoprenylcysteine carboxyl methyltransferase & -2.05 & 0.59 \\
\hline 209897_s_at & SLIT2 & Slit homolog 2 (Drosophila) & -2.05 & 0.42 \\
\hline 215464_s_at & TAX1BP3 & Tax1 (human T-cell leukemia virus type I) binding protein 3 & -2.04 & 0.56 \\
\hline 200922_at & KDELR1 & KDEL (Lys-Asp-Glu-Leu) endoplasmic reticulum protein retention receptor 1 & -2.04 & 0.58 \\
\hline 204094_s_at & TSC22D 2 & TSC22 domain family, member 2 & -2.04 & 0.61 \\
\hline 209440_at & PRPS1 & Phosphoribosyl pyrophosphate synthetase 1 & -2.04 & 0.61 \\
\hline 201729_s_at & KIAA0100 & KIAA0100 & -2.03 & 0.47 \\
\hline 204117_at & PREP & Prolyl endopeptidase & -2.03 & 0.60 \\
\hline 200885_at & PPM1J & Protein phosphatase 1J (PP2C domain containing) & -2.03 & 0.64 \\
\hline 200869_at & RPL18A & Ribosomal protein L18a & -2.02 & 0.63 \\
\hline 218384_at & CARHSP1 & Calcium regulated heat stable protein $1,24 \mathrm{kDa}$ & -2.01 & 0.60 \\
\hline 221039_s_at & DDEF1 & Development and differentiation enhancing factor 1 & -2.01 & 0.58 \\
\hline 218618_s_at & FNDC3B & Fibronectin type III domain containing 3B & -2.01 & 0.33 \\
\hline 200752_s_at & CAPN1 & Calpain $1,(\mathrm{mu} / \mathrm{I})$ large subunit & -1.99 & 0.57 \\
\hline 212669_at & CAMK2G & Calcium/calmodulin-dependent protein kinase (CaM kinase) II $\gamma$ & -1.97 & 0.62 \\
\hline 200008_s_at & GDI2 & GDP dissociation inhibitor 2 & -1.97 & 0.64 \\
\hline 203931_s_at & MRPL12 & Mitochondrial ribosomal protein L12 & -1.97 & 0.64 \\
\hline
\end{tabular}


Table II. Continued.

\begin{tabular}{|c|c|c|c|c|}
\hline Probe & Symbol & Description $^{\mathrm{a}}$ & Score $^{b}$ & $\begin{array}{c}\text { Fold } \\
\text { change }^{\mathrm{b}}\end{array}$ \\
\hline 221699_s_at & DDX50 & DEAD (Asp-Glu-Ala-Asp) box polypeptide 50 & -1.97 & 0.67 \\
\hline 222162_s_at & ADAMTS1 & ADAM metallopeptidase with thrombospondin type 1 motif, 1 & -1.97 & 0.59 \\
\hline 212363_x_at & ACTG1 & Actin, $\gamma 1$ & -1.96 & 0.70 \\
\hline 204275_at & SOLH & Small optic lobes homolog (Drosophila) & -1.96 & 0.62 \\
\hline 55705_at & FMN2 & Formin 2 & -1.95 & 0.68 \\
\hline 32811_at & MYO1C & Myosin IC & -1.95 & 0.70 \\
\hline 217234_s_at & VIL2 & Villin 2 (ezrin) & -1.95 & 0.60 \\
\hline 218496_at & RNASEH1 & Ribonuclease $\mathrm{H} 1$ & -1.94 & 0.58 \\
\hline 210658_s_at & GGA2 & Golgi associated, $\gamma$ adaptin ear containing, ARF binding protein 2 & -1.949 & 0.60 \\
\hline 210555_s_at & NFATC3 & Nuclear factor of activated T-cells, cytoplasmic, calcineurin-dependent 3 & -1.93 & 0.61 \\
\hline 215446_s_at & LOX & Lysyl oxidase & -1.91 & 0.68 \\
\hline 201143_s_at & EIF2S1 & Eukaryotic translation initiation factor 2 , subunit $1 \alpha, 35 \mathrm{kDa}$ & -1.90 & 0.61 \\
\hline 210125_s_at & BANF1 & Barrier to autointegration factor 1 & -1.89 & 0.61 \\
\hline 204875_s_at & GMDS & GDP-mannose 4,6-dehydratase & -1.89 & 0.64 \\
\hline 209391_at & DPM2 & Dolichyl-phosphate mannosyltransferase polypeptide 2 , regulatory subunit & -1.89 & 0.62 \\
\hline 216602_s_at & FARSLA & Phenylalanine-tRNA synthetase-like, $\alpha$ subunit & -1.88 & 0.60 \\
\hline 40562_at & GNA11 & Guanine nucleotide binding protein (G protein), $\alpha 11$ (Gq class) & -1.88 & 0.64 \\
\hline 213509_x_at & CES2 & Carboxylesterase 2 (intestine, liver) & -1.88 & 0.68 \\
\hline 201516_at & SRM & Spermidine synthase & -1.88 & 0.68 \\
\hline 218088_s_at & RRAGC & Ras-related GTP binding C & -1.87 & 0.62 \\
\hline 211271_x_at & PTBP1 & Polypyrimidine tract binding protein 1 & -1.87 & 0.66 \\
\hline 209100_at & IFRD2 & Interferon-related developmental regulator 2 & -1.85 & 0.67 \\
\hline 208336_s_at & GPSN2 & Glycoprotein, synaptic 2 & -1.84 & 0.59 \\
\hline 221693_s_at & MRPS18A & Mitochondrial ribosomal protein S18A & -1.83 & 0.66 \\
\hline 208623_s_at & VIL2 & Villin 2 (ezrin) & -1.80 & 0.64 \\
\hline 208996_s_at & POLR2C & Polymerase (RNA) II (DNA directed) polypeptide C, $33 \mathrm{kDa}$ & -1.79 & 0.68 \\
\hline 212586_at & CAST & Calpastatin & -1.78 & 0.73 \\
\hline 208991_at & STAT3 & Signal transducer and activator of transcription 3 (acute-phase response factor) & -1.78 & 0.67 \\
\hline 201979_s_at & PPP5C & Protein phosphatase 5 , catalytic subunit & -1.75 & 0.66 \\
\hline 210978_s_at & TAGLN2 & Transgelin 2 & -1.72 & 0.69 \\
\hline 201667_at & GJA1 & Gap junction protein, alpha $1,43 \mathrm{kDa}$ (connexin 43 ) & -1.71 & 0.72 \\
\hline 219068_x_at & ATAD3A & ATPase family, AAA domain containing 3A & -1.71 & 0.69 \\
\hline 208677_s_at & BSG & Basigin (Ok blood group) & -1.69 & 0.69 \\
\hline 218001_at & MRPS2 & Mitochondrial ribosomal protein S2 & -1.67 & 0.72 \\
\hline 200991_s_at & SNX17 & Sorting nexin 17 & -1.63 & 0.73 \\
\hline 200638_s_at & YWHAZ & $\begin{array}{l}\text { Tyrosine } 3 \text {-monooxygenase/tryptophan } 5 \text {-monooxygenase activation protein, } \\
\zeta \text { polypeptide }\end{array}$ & -1.63 & 0.73 \\
\hline 205178_s_at & RBBP6 & Retinoblastoma binding protein 6 & -1.62 & 0.71 \\
\hline 211026_s_at & MGLL & Monoglyceride lipase & -1.61 & 0.74 \\
\hline 212131_at & LSM14A & LSM14 homolog A (SCD6, S. cerevisiae) & -1.59 & 0.73 \\
\hline 203342_at & TIMM17B & Translocase of inner mitochondrial membrane 17 homolog B (yeast) & -1.47 & 0.76 \\
\hline
\end{tabular}

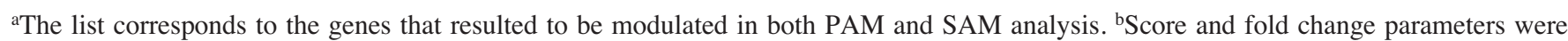
obtained by SAM analysis.

regulate transcription, protein modification/biosynthesis and RNA processing/metabolism (Table III). Only five of the down-modulated genes resulted to be involved in the angiogenic process: SDC-4, Id-1 and Id-3, tumor necrosis factor receptor superfamily member $12 \mathrm{~A}$ (TNFRSF12A) and nuclear factor of activated T-cells C3 (NFATC3) (Tables III and IV). The protein inhibitor of activated STAT3 (PIAS3) was the only up-regulated gene involved in angiogenesis (Table III and IV).
Effect of GPI 15427 on SDC-4 and Id-1 expression. The influence of treatment with GPI 15427 on SDC-4 and Id-1 expression in endothelial cells was further studied at the protein level by Western blot analysis (Fig. 1). The results indicated that SDC-4 protein was strongly reduced (61 to $67 \%$ inhibition) in treated cells at short incubation times after drug exposure (2 and $6 \mathrm{~h}$ ). At later time points, SDC-4 expression was up-regulated during the culture both in untreated or GPI 15427-treated endothelial cells. On the other 
Table III. Functional analysis of genes modulated after GPI 15427 treatment of endothelial cells.

\begin{tabular}{|c|c|c|}
\hline Biological process ${ }^{\mathrm{a}}$ & Genes & Percentag \\
\hline Regulation of transcription & $\begin{array}{l}\text { STAT3 Id-3 SMARCC1 SERTAD2 PNN NFATC3 NR3C1 } \\
\text { BNC2 CRK CSDE1 ID1 CLOCK CARHSP1 RPS14 PAWR } \\
\text { SCAND1 HMG2L1 TSC22D2 LRRFIP1 ZNF580 PIAS3 }\end{array}$ & 19.8 \\
\hline Protein modification & $\begin{array}{l}\text { LOX PPP5C DPM2 ICMT PPM1J CAMK2G PSENEN } \\
\text { SPOP UBE2M RBBP6 GNA11 MTMR4 STT3A UBE2G2 } \\
\text { CTBP1 PRMT1 RNF126 PIAS3 }\end{array}$ & 17.0 \\
\hline Protein biosynthesis & $\begin{array}{l}\text { MRPL12 DPM2 MRPS18A EIF3S4 EIF2S3 RPL18A } \\
\text { PUM1 EIF2S1 RPS14 PAWR STT3A FARSLA } \\
\text { EIF4G1 EIF5A MRPS2 }\end{array}$ & 14.1 \\
\hline $\begin{array}{l}\text { RNA processing } \\
\text { and mRNA metabolism }\end{array}$ & $\begin{array}{l}\text { RRAGC PTBP1 PNN DIMT1L SPOP RPS14 PRPF31 } \\
\text { MAGOH PCBP2 SF3A2 }\end{array}$ & 9.4 \\
\hline Protein targeting & YWHAZ ICMT SEC61A1 TIMM17B CDC37 TIMM44 & 5.7 \\
\hline Proteolysis & SOLH ADAMTS1 PREP CAPN1 PSENEN UBE2G2 & 5.7 \\
\hline Angiogenesis & Id-1 Id-3 NFATC3 SDC-4 TNFRSF12A PIAS3 & 5.7 \\
\hline Actin filament-based process & ACTG1 CRK FLNA FMN2 VIL2 GSN & 5.7 \\
\hline Protein folding & PPIB DNAJC7 CDC37 PFDN6 & 3.8 \\
\hline Glycoprotein metabolism & DPM2 PSENEN PAWR STT3A & 3.8 \\
\hline
\end{tabular}

a Regulated biological processes were identified by the GEPAS web-based application according to the Gene Ontology Consortium classification. ${ }^{\mathrm{b}}$ The values indicate the percentage of genes that have been assigned to each biological process among the total number of genes modulated by treatment of endothelial cells with GPI 15427 .

Table IV. Genes known to be involved in the angiogenic process that are modulated by treatment of endothelial cells with GPI 15427.

\begin{tabular}{|c|c|c|c|c|}
\hline Probe & Symbol & Description & Score $^{\mathrm{a}}$ & Fold change $^{a}$ \\
\hline 203035_s_at & PIAS3 & Protein inhibitor of activated STAT, 3 & 3.21 & 1.85 \\
\hline 202071_at & SDC-4 & Syndecan 4 (amphiglycan, ryudocan) & -4.56 & 0.41 \\
\hline 208937_s_at & Id-1 & Inhibitor of DNA binding 1, dominant negative helix-loop-helix protein & -4.34 & 0.22 \\
\hline 218368_s_at & TNFRSF12A & Tumor necrosis factor receptor superfamily, member $12 \mathrm{~A}$ & -2.45 & 0.43 \\
\hline 207826_s_at & Id-3 & Inhibitor of DNA binding 3, dominant negative helix-loop-helix protein & -2.31 & 0.45 \\
\hline 210555_s_at & NFATC3 & Nuclear factor of activated T-cells, cytoplasmic, calcineurin-dependent 3 & -1.93 & 0.61 \\
\hline
\end{tabular}

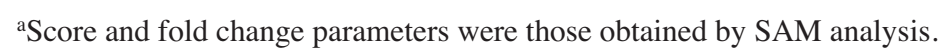

hand, Id-1 protein expression started to be down-modulated only 6 to $24 \mathrm{~h}$ after treatment, with a $55 \%$ reduction in treated cells with respect to the untreated control at $30 \mathrm{~h}$. The decrease of Id-1 protein expression occurred later with respect to that of the corresponding mRNA transcription which, instead, was detected $2 \mathrm{~h}$ after treatment with the PARP inhibitor. Experiments performed to analyze basal expression of Id-1 in HUV-ST cells indicated that this protein was absent in the cell extracts prepared from cultures grown in medium lacking growth factor supplements (data not shown).
Effect of GPI 15427 on transcription factor activity. Gene functional analysis showed that the majority of modulated genes, including most of those involved in angiogenesis, are under the control of the transcription factor NF- $\mathrm{KB}$, which is known to be regulated by PARP-1 (Table V). Other transcription factors known to be regulated by PARP-1 are indicated in Table V. Among them, Oct-1 regulates $13 \%$ of the genes down-modulated by GPI 15427 treatment (including SDC-4), while AP-1 modulates $6.2 \%$ of the genes (but none of them related to angiogenesis). Only one gene, affected by 
A
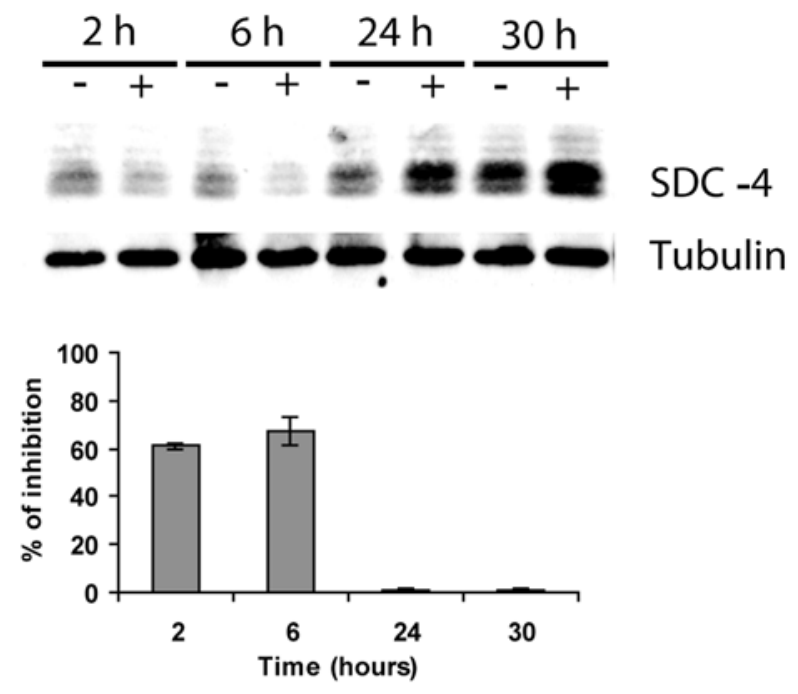

B


Figure 1. Effect of GPI 15427 on SDC-4 and Id-1 expression. HUV-ST cells, untreated or incubated with $2 \mu \mathrm{M}$ GPI 15427 for the indicated periods of time, were analyzed for the expression of SDC-4 (A) and Id-1 (B) polypeptides by Western blotting. Blots are representative of 1 out of 3 independent experiments with similar results. SDC-4 and Id-1 proteins were quantified by densitometric analysis followed by normalization against the B-tubulin content of the samples. Histograms represent the mean $( \pm$ SD) percentage inhibition of SDC-4 or Id-1 expression in GPI 15427-treated cells with respect to untreated control using the data from 3 independent experiments.

treatment with the PARP inhibitor, but not related to angiogenesis, is under the control of HIF-1. Interestingly, the members of the activating transcription factor family c-AMP response element binding protein (CREB)/ATF-1 and ATF-3 regulate the transcription of Id- 1 (Table V).

The activity and/or expression of transcription factors that might be involved in the effect of GPI 15427 on the angiogenic capabilities of human endothelial cells were tested. The results indicated that in the endothelial cells used for the present study the NF- $\mathrm{KB}$ activity was very low and was not modulated by
A

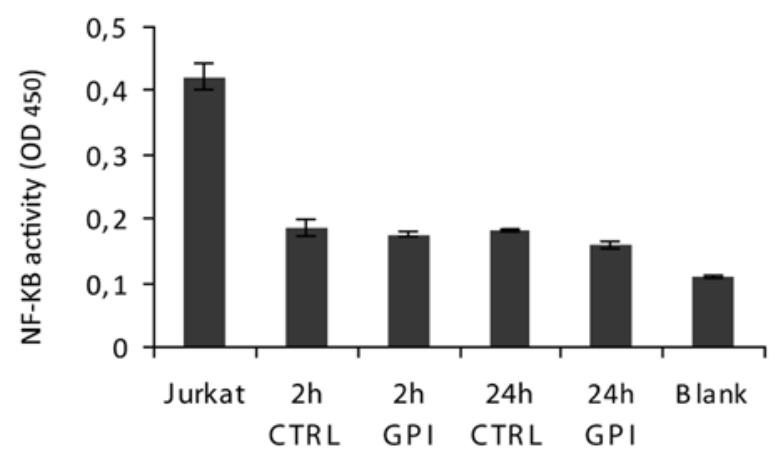

B

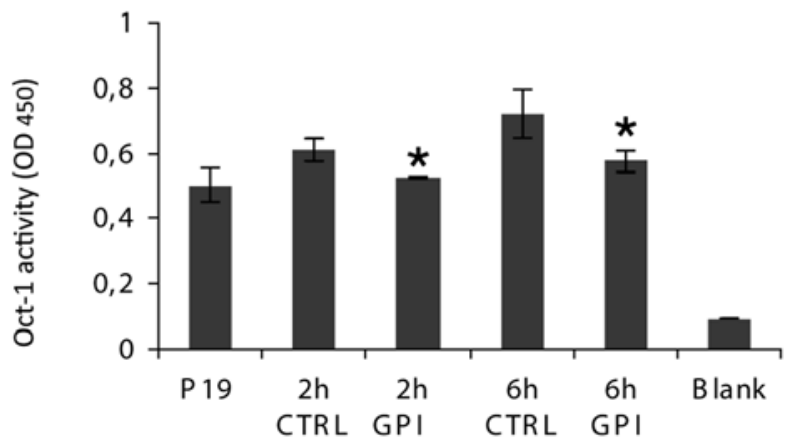

Figure 2. Effect of GPI 15427 on NF-кB and Oct-1 transcription factors Nuclear extracts prepared from HUV-ST cells, untreated or incubated with $2 \mu \mathrm{M}$ GPI 15427 for the indicated periods of time, were analyzed for the activity of NF- $\mathrm{\kappa B}$ (A) or Oct-1 (B) as described in Materials and methods using a TransAm kit. Data are representative of 1 out of 3 independent experiments with similar results. Bars indicate the SD of the mean. A) Statistical analysis of NF-кB activity in control versus GPI 15427- treated groups by Student's t test: not significant. B) Statistical analysis of Oct-1 activity in control versus GPI 15427-treated groups by Student's t test, ${ }^{*} \mathrm{P}<0.05$.

the PARP inhibitor (Fig. 2A). In the conditions tested, Oct-1 activity resulted to be only slightly, but significantly, downmodulated (up to $16 \%$ ) by treatment with the inhibitor for 2 or $6 \mathrm{~h}$ (Fig. 2B). Oct-1 protein is abundantly produced in HUV-ST cells, but its expression was not substantially modulated by GPI 15427 treatment (data not shown), suggesting that PARP-1 could directly modulate Oct- 1 activity.

Analysis of CREB/ATF-1 levels and phosphorylation showed that both proteins were modulated during the period of culture analyzed, being barely detectable few hours after the beginning of culture and up-regulated at later time points. In the case of ATF-1, protein and phosphorylation levels were almost unaffected by the drug treatment (Fig. 3). On the other hand, up-regulation of CREB expression was blocked by the PARP inhibitor and this effect was paralleled by a reduced phosphorylation of the protein. In regard to ATF-3, no changes in the level of protein expression were observed and a modest decrease of phosphorylation of this transcription factor was detected in the first $2 \mathrm{~h}$ (data not shown). 
Table V. Genes modulated after GPI 15427 treatment of endothelial cells that are under the control of specific transcription factors regulated by PARP-1.

\begin{tabular}{|c|c|c|}
\hline Transcription factors ${ }^{\mathrm{a}}$ & Genes & Percentage $^{b}$ \\
\hline $\mathrm{NF}-\kappa \mathrm{B}$ & $\begin{array}{l}\text { FNDC3B MYO1C GLT25D1 GNAI2 Id-3 PLD3 PPP5C } \\
\text { WDR68 GPSN2 NUCKS1 NFATC3 NR3C1 BNC2 SEC61A1 } \\
\text { DIMT1L GDI2 SDC-4 EIF2S3 CAMK2G PRPS1 CAPN1 } \\
\text { PSENEN FMN2 UBE2M ARF1 DDX50 CDC37 MTMR4 } \\
\text { FARSLA CXADR EIF4G1 PRMT1 DAP TSPAN4 HMG2L1 } \\
\text { NDUFB7 TXNRD2 SERF2 HLA-C GSN PIAS3 HLA-B }\end{array}$ & 28.8 \\
\hline Oct-1 & $\begin{array}{l}\text { STAT3 SETD5 RHOBTB3 SMARCC1 MRPS18A CRK } \\
\text { SDC-4 EIF2S3 PRPS1 CLOCK SRM BANF1 SCAND1 } \\
\text { PRMT1 HMG2L1 NDUFB7 SLC29A1 TSC22D2 GSN }\end{array}$ & 13.0 \\
\hline AP-1 & $\begin{array}{l}\text { GMDS BNC2 IFRD2 CAMK2G PRPS1 KIAA0100 SPOP } \\
\text { TIMM44 RAB3B }\end{array}$ & 6.2 \\
\hline CREB/ATF-1 & GLT25D1 STAT3 Id-1 AP2B1 RBBP6 CDC37 DAP TSC22D2 & 5.5 \\
\hline ATF3 & STAT3 IFRD2 Id-1 AP2B1 RBBP6 DAP TSC22D2 & 4.8 \\
\hline ATF4 & SOLH STAT3 Id-3 AP2B1 TIMM44 NDUFB7 TSC22D2 & 4.8 \\
\hline ATF6 & STAT3 RBBP6 CTBP1 & 2.0 \\
\hline HIF-1 & CDT1 & 0.7 \\
\hline
\end{tabular}

\section{Discussion}

Following our recent publication on the role of PARP-1 in angiogenesis and on the impairment of endothelial functions mediated by PARP inhibition with GPI 15427 (10), we investigated the early molecular mechanisms involved in this effect analyzing the modulation of gene expression in endothelial cells treated with non-toxic concentrations of the PARP inhibitor. In the present study we demonstrate, for the first time, that SDC-4 and Id-1 proteins, which are both involved in angiogenesis, are down-regulated by GPI 15427 treatment.

Treatment of endothelial cells with the PARP inhibitor for $2 \mathrm{~h}$ provoked a prompt decrease of SDC- 4 both at the transcript and protein level. SDC-4 is the most ubiquitously expressed member of a transmembrane heparin sulfate proteoglycans family that binds to and can be activated by insoluble ligands, such as extracellular matrix molecules, and soluble ligands, including growth factors (21). Moreover, SDC-4 is activated by angiogenic factors and is involved in endothelial cell adhesion and migration $(22,23)$. The effect of the treatment with the PARP inhibitor on SDC-4 expression in endothelial cells was transient, since $24 \mathrm{~h}$ after treatment the decrease of this protein was no longer detectable. Therefore, it is possible to hypothesize that SDC-4 protein might be required in the initial steps of the angiogenesis process, such as the triggering of endothelial cell migration from the pre-existing vessel, that is compatible with the known role of this protein in endothelial cell adhesion and migration $(22,23)$.
The Id-1 protein was down-regulated by GPI 15427 at a later time point with respect to SDC-4. Id-1 is a member of the helix-loop-helix family of proteins that does not contain DNA binding sequences but prevents transcription factors from binding to DNA by direct physical interaction (24). Id-1 plays a key role in regulating angiogenesis, including that associated with tumor development (25). The expression of this protein can be induced in endothelial cells by VEGF and TGF, and its overexpression enhances I-CAM-1 and Eselectin expression, and provokes transmigration, matrix metalloproteinase production and tube formation (26). Knockdown of Id-1 in endothelial cells abolished proliferation and angiogenic processes induced by VEGF (26). Induction of the Id-1 protein by VEGF supports the hypothesis that the previously observed effects of GPI 15427 on VEGF-induced angiogenic processes (10) might indeed involve this protein.

A short exposure of endothelial cells to the PARP inhibitor resulted in transcription down-regulation of a limited number of genes known to play a role in angiogenesis. While SDC-4 and Id- 1 were found to be the mostly affected genes, the other genes found to be inhibited were TNTRSF12A, a member of the TNF receptor family which interacts with TNF-like weak inducer of apoptosis (TWEAK) (27), Id-3, another member of the helix-loop-helix family of proteins $(25,26)$ and NFATC3, a transcription factor belonging to a protein family controlled by calcineurin which regulates vascular development during angiogenesis (28). Among the up-regulated genes, PIAS3 was the most significantly up-regulated. PIAS3 is a negative 
A

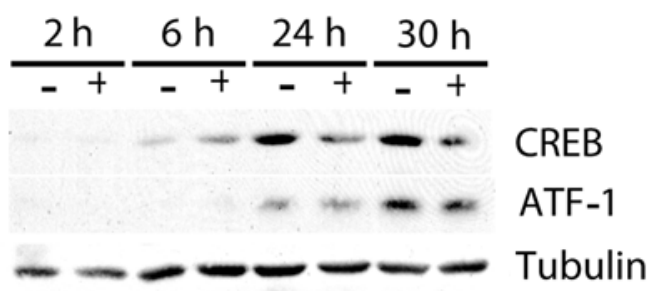

B

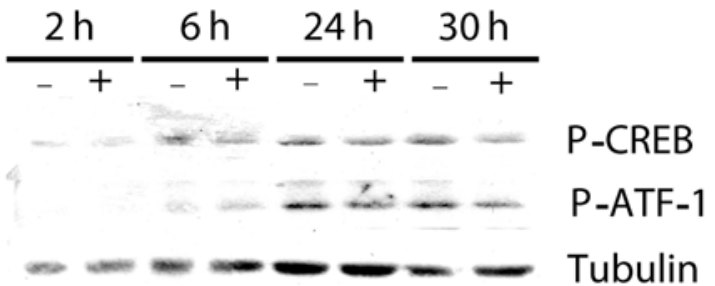

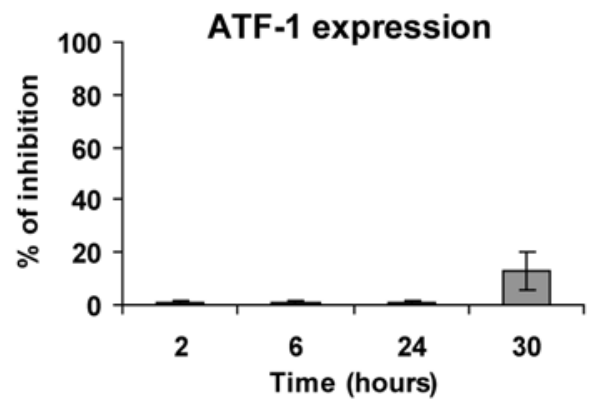

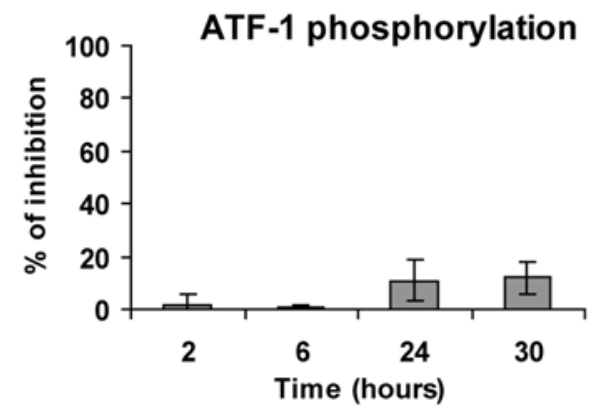

Figure 3. Effect of GPI 15427 on CREB and ATF-1 activation. Cell extracts were prepared at the indicated time points and analyzed by Western blotting for the presence of total (A) or phosphorylated (B) CREB and ATF-1 proteins. Results were quantified by densitometric analysis as indicated in the legend of Fig. 1. Blots are representative of 1 out of 3 independent experiments with similar results and histograms represent the mean ( \pm SD) percentage inhibition of the protein or phosphoprotein analyzed in GPI 15427-treated cells with respect to untreated control.

regulator of STAT3, a transcription factor involved in VEGF expression $(29,30)$. However, in HUV-ST cells or in HUVEC the levels of VEGF released by the cells was extremely low and was not modulated by GPI 15427 treatment (10).

Microarray analysis revealed a prevalent down-modulation of gene expression, the highest percentage of which is involved in regulation of gene transcription. It is well known that PARP-1 regulates transcription by at least two different mechanisms: modulating chromatin structure and acting as part of gene-specific enhancer/promoter-binding complexes (31). This activity is accomplished by PARP-1 through a direct protein-protein interaction or through covalent poly(ADPribosyl)ation of the target molecule (32). For instance, poly(ADP-ribosyl)ation of histones loosens chromatin and renders genes accessible to the transcriptional machinery. Moreover, non-covalent interaction of histones with poly (ADP-ribose), as free polymer or attached to proteins, may also allow release of histones from nucleosome rendering DNA accessible for transcription (32-34). Even though in cells not subjected to DNA damage the average length of ADP-ribose polymers is shorter than in cells exposed to genotoxic injury, ADP-ribose polymers may still participate to the regulation of several functions also in intact cells. In addition, PARP-1 interacts with a number of transcription factors such as NF-кB, p53, Oct-1, B-MYB, AP-1, HIF-1, acting as transcriptional co-regulator (32-37). It is likely that the reduction of gene transcription induced by GPI 15427 might be the result of a decrease of cellular ADP-ribose polymers content rather than of compromised protein interactions with PARP-1. In fact, similarly to the other PARP inhibitors presently available, GPI 15427 targets the NAD ${ }^{+}$binding domain of the enzyme hampering the use of $\mathrm{NAD}^{+}$as substrate for the synthesis of ADP-ribose polymers (16).

Gene functional analysis, performed according to the Gene Ontology Consortium classification, showed that about $28 \%$ of the genes (including Id-3, NFATC3, SDC-4 and PIAS3 which are involved in angiogenesis) modulated by GPI 15427 treatment of endothelial cells were under the control 


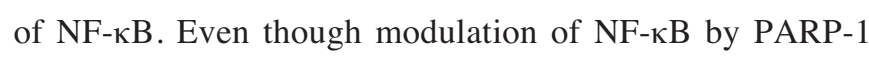
presumably requires direct protein-protein interaction, it has been shown that $\mathrm{NF}-\kappa \mathrm{B}$ is capable of binding poly(ADPribose), indicating that its activity may be regulated also by non-covalent interaction with the polymer (38). Nevertheless, our results showed that in the endothelial cell model used NF- $\mathrm{\kappa B}$ activity was barely detectable and was not modulated by treatment with the PARP inhibitor GPI 15427. Also HIF-1 $\alpha$ was not found to be modulated by GPI 15427 in this model of endothelial cells (10). Gene functional analysis showed that the transcription of $13 \%$ of down-regulated genes (including SDC-4) is controlled by the octamer trans-acting factor Oct-1, a transcription regulator capable of interacting with PARP-1, which stabilizes its binding to the DNA octamer motif (39). This transcription factor was expressed by HUV-ST cells and its activity was slightly down-modulated by GPI 15427 in the assay used. Nevertheless, it cannot be excluded that the druginduced reduction of Oct-1 activity might be underestimated. In fact, it is likely that the physical interaction between Oct-1 and PARP-1 might not be fully maintained during the preparation of the nuclear extracts or during the in vitro activity assay.

PARP-1 has also been involved in the modulation of the ATF family of transcription factors, favoring phosphorylation of these proteins (40). Functional analysis of genes modulated by GPI 15427 in endothelial cells indicated that members of the ATF protein family (ATF-1, CREB and ATF-3) regulate Ids gene expression. In particular, it has been shown in normal human keratinocytes that Ids transcription can be modulated through cis-acting elements in the distal portion of the promoter, including a CREB-binding site (41). Therefore, it is possible that the strong down-modulation of activated CREB protein after $6 \mathrm{~h}$ of treatment would contribute to the reduction in Id-1 protein expression. The delayed decrease of Id-1 protein with respect to the prompt reduction of mRNA expression might depend on the half-life of the previously synthesized protein. As described by Ling and colleagues, the half-life of Id-1 protein is quite variable depending on cell type (42). We observed that the increased stability of the protein appears to be a characteristic of endothelial cells maintained in medium with a mix of growth factors (bFGF, VEGF, EGF and IGF-1) (data not shown), some of which are known to sustain Id-1 expression (26). Finally, the modest decrease of ATF-3 phosphorylation detected $2 \mathrm{~h}$ after treatment is unlikely to account for the reduction of Id-1 transcript since ATF-3 has been found to be a negative regulator of this protein (43). Therefore, the initial inhibition of Id-1 transcript during the first $2 \mathrm{~h}$ of treatment with the PARP inhibitor might be due to the effect of the drug on other still undefined molecular mechanisms.

Previous studies have analyzed the effects of PARP-1 deficiency on gene expression at the genome wide-level, using murine PARP $-1 \%$ embryonic stem cell lines and cells of different tissue origin $(37,44,45)$ or cells derived from PARP- $1^{+/+}$mice treated with a PARP inhibitor (37). The lack of PARP-1 expression was associated with both downregulation and up-regulation of genes involved in a variety of processes such as metabolism, signal transduction, cell cycle control and transcription, which varied depending on the cell system investigated (44). In skin-derived cells obtained from
$\mathrm{P}-1^{-/}$mice or from PARP $-1^{+/+}$mice treated with the PARP inhibitor 3,4-dihydro-5-[4-(1-piperidinyl)butoxyl]-1(2H)isoquinolinone (DPQ), differences were found in genes involved in carcinogenesis and inflammation with respect to controls (37). In regard to endothelial cells, gene expression profiles in response to TNF- $\alpha$ treatment were investigated in cells derived from the heart vasculature of PARP-1/- mice and the absence of PARP-1 was found to hamper the expression of genes up-regulated during the inflammatory process (45). However, since no studies have been performed to date on human endothelial cells, it is difficult to make comparisons between our results and the previously published studies, due to the differences in the species, tissue origin and stimuli used.

In conclusion, treatment of human endothelial cells with the potent PARP inhibitor GPI 15427 induced a strong downmodulation of at least two gene products that play an important role in endothelial functions: SDC-4 and Id-1. It can be hypothesized that the mechanism by which GPI 15427 exerts the inhibitory effect on angiogenesis (10), might be ascribed to a reduced PARP-mediated regulation of the activity of certain transcription factors that control endothelial functions. In this context, inhibition of Oct-1 and CREB/ATF family of transcription factors might be crucial in the down-modulation of molecules involved in endothelial cell migration, adhesion, proliferation or differentiation during the angiogenic process.

\section{Acknowledgements}

This study was supported by grants from the Italian Ministry of Education and Research, 'Programmi di Ricerca scientifica di rilevante Interesse Nazionale' (PRIN) projects to G.G. and by the Italian Ministry of Health (RC07-3.16 project to P.M.L.). We would like to thank Mauro H. Citterich for his technical assistance in the hybridization of the GeneChip microarray.

\section{References}

1. Hassa PO and Hottiger MO: The diverse biological roles of mammalian PARPS, a small but powerful family of poly-ADPribose polymerases. Front Biosci 13: 3046-3082, 2008.

2. Yélamos J, Schreiber V and Dantzer F: Toward specific functions of poly(ADP-ribose) polymerase-2. Trends Mol Med 14: 169-178, 2008.

3. Schreiber V, Amé JC, Dollé P, et al: Poly(ADP-ribose) polymerase-2 (PARP-2) is required for efficient base excision DNA repair in association with PARP-1 and XRCC1. J Biol Chem 277: 23028-23036, 2002.

4. Tentori L and Graziani G: Chemopotentiation by PARP inhibitors in cancer therapy. Pharmacol Res 52: 25-33, 2005

5. Galande S and Kohwi-Shigematsu T: Poly(ADP-ribose) polymerase and $\mathrm{Ku}$ autoantigen form a complex and synergistically bind to matrix attachment sequences. J Biol Chem 274: 20521-20528, 1999.

6. Malanga $\mathrm{M}$ and Althaus FR: Poly(ADP-ribose) reactivates stalled DNA topoisomerase I and induces DNA strand break resealing. J Biol Chem 279: 5244-5248, 2004.

7. Ghodgaonkar MM, Zacal N, Kassam S, Rainbow AJ and Shah GM: Depletion of poly(ADP-ribose) polymerase-1 reduces host cell reactivation of a UV-damaged adenovirusencoded reporter gene in human dermal fibroblasts. DNA Repair 7: 617-632, 2008.

8. Farmer H, McCabe N, Lord CJ, et al: Targeting the DNA repair defect in BRCA mutant cells as a therapeutic strategy. Nature 434: 917-921, 2005.

9. Bryant HE, Schultz N, Thomas HD, et al: Specific killing of BRCA2-deficient tumours with inhibitors of poly(ADP-ribose) polymerase. Nature 434: 913-917, 2005. 
10. Tentori L, Lacal PM, Muzi A, et al: Poly(ADP-ribose) polymerase (PARP) inhibition or PARP-1 gene deletion reduces angiogenesis. Eur J Cancer 43: 2124-2133, 2007.

11. Rajesh M, Mukhopadhyay P, Godlewski G, Bátkai S, Haskó G, Liaudet L and Pacher P: Poly(ADP-ribose)polymerase inhibition decreases angiogenesis. Biochem Biophys Res Commun 350: 1056-1062, 2006.

12. Rajesh M, Mukhopadhyay P, Bátkai S, Godlewski G, Haskó G, Liaudet L and Pacher P: Pharmacological inhibition of poly (ADP-ribose) polymerase inhibits angiogenesis. Biochem Biophys Res Commun 350: 352-357, 2006.

13. Pyriochou A, Olah G, Deitch EA, Szabó C and Papapetropoulos A: Inhibition of angiogenesis by the poly(ADP-ribose) polymerase inhibitor PJ-34. Int J Mol Med 22: 113-118, 2008.

14. Tentori L, Muzi A, Dorio AS, et al: Stable depletion of poly (ADP-ribose) polymerase-1 reduces in vivo melanoma growth and increases chemosensitivity. Eur J Cancer 44: 1302-1314, 2008.

15. Tentori L, Vergati M, Muzi A, et al: Generation of an immortalized human endothelial cell line as a model of neovascular proliferating endothelial cells to assess chemosensitivity to anticancer drugs. Int J Oncol 27: 525-535, 2005.

16. Tentori L, Leonetti C, Scarsella M, et al: Systemic administration of GPI 15427, a novel poly(ADP-ribose) polymerase-1 inhibitor increases the antitumor activity of temozolomide against intracranial melanoma, glioma, lymphoma. Clin Cancer Res 9: 5370-5379, 2003.

17. Bakondi E, Bai P, Szabo EE, Hunyadi J, Gergely P, Szabo C and Virag L: Detection of poly(ADP-ribose) polymerase activation in oxidatively stressed cells and tissues using biotinylated NAD substrate. J Histochem Cytochem 50: 91-98, 2002.

18. Tibshirani R, Hastie T, Narasimhan B and Chu G: Diagnosis of multiple cancer types by shrunken centroids of gene expression. Proc Natl Acad Sci USA 99: 6567-6572, 2002.

19. Gentleman R and Carey VJ: Visualization and annotation of genomic experiments. In: The Analysis of Gene Expression Data. Parmigiani G, Garrett ES, Irizarry RA and Zeger SL (eds). Springer, New York, pp46-72, 2003.

20. Al-Shahrour F, Minguez P, Tárraga J, et al: BABELOMICS: a systems biology perspective in the functional annotation of genome-scale experiments. Nucleic Acids Res 34: W472-W476, 2006.

21. Bernfield M, Götte M, Park PW, Reizes O, Fitzgerald ML, Lincecum $\mathrm{J}$ and Zako M: Functions of cell surface heparan sulfate proteoglycans. Annu Rev Biochem 68: 729-777, 1999.

22. Tkachenko E, Elfenbein A, Tirziu D and Simons M: Syndecan-4 clustering induces cell migration in a PDZ-dependent manner. Circ Res 98: 1398-1404, 2006.

23. Nunes SS, Outeiro-Bernstein MA, Juliano L, et al: Syndecan-4 contributes to endothelial tubulogenesis through interactions with two motifs inside the pro-angiogenic $\mathrm{N}$-terminal domain of thrombospondin-1. J Cell Physiol 214: 828-837, 2008.

24. Benezra R, Rafii S and Lyden D: The Id proteins and angiogenesis. Oncogene 20: 8334-8341, 2001.

25. Nishiyama $K$, Takaji K, Kataoka K, et al: Id1 gene transfer confers angiogenic property on fully differentiated endothelial cells and contributes to therapeutic angiogenesis. Circulation 112: 2840-2850, 2005.

26. Sakurai D, Tsuchiya N, Yamaguchi A, et al: Crucial role of inhibitor of DNA binding/differentiation in the vascular endothelial growth factor-induced activation and angiogenic processes of human endothelial cells. J Immunol 173: 5801-5809, 2004.

27. Wiley SR, Cassiano L, Lofton T, et al: A novel TNF receptor family member binds TWEAK and is implicated in angiogenesis. Immunity 15: 837-846, 2001.
28. Horsley V and Pavlath GK: NFAT: ubiquitous regulator of cell differentiation and adaptation. J Cell Biol 156: 771-774, 2002

29. Chung CD, Liao J, Liu B, Rao X, Jay P, Berta P and Shuai K: Specific inhibition of Stat3 signal transduction by PIAS3. Science 278: 1803-1805, 1997

30. Bartoli M, Platt D, Lemtalsi T, Gu X, Brooks SE, Marrero MB and Caldwell RB: VEGF differentially activates STAT3 in microvascular endothelial cells. FASEB J 17: 1562-1564, 2003.

31. Aguilar-Quesada R, Muñoz-Gámez JA, Martín-Oliva D, et al: Modulation of transcription by PARP-1: consequences in carcinogenesis and inflammation. Curr Med Chem 14: 1179-1187, 2007.

32. Kraus WL and Lis JT: PARP goes transcription. Cell 113: 677-683, 2003

33. Malanga M, Pleschke JM, Kleczkowska HE and Althaus FR: Poly(ADP-ribose) binds to specific domains of p53 and alters its DNA binding functions. J Biol Chem 273: 11839-11843, 1998.

34. Malanga $\mathrm{M}$ and Althaus FR: The role of poly(ADP-ribose) in the DNA damage signaling network. Biochem Cell Biol 83: 354-364, 2005.

35. Jagtap P and Szabo C: Poly(ADP-ribose) polymerase and the therapeutic effects of its inhibitors. Nat Rev Drug Discov 4: 421-440, 2005.

36. Andreone TL, O'Connor M, Denenberg A, Hake PW and Zingarelli B: Poly(ADP-ribose) polymerase-1 regulates activation of activator protein-1 in murine fibroblasts. $J$ Immunol 170: 2113-2120, 2003.

37. Martin-Oliva D, Aguilar-Quesada R, O'valle F, et al: Inhibition of poly(ADP-ribose) polymerase modulates tumor-related gene expression, including hypoxia-inducible factor-1 activation, during skin carcinogenesis. Cancer Res 66: 5744-5756, 2006.

38. Pleschke JM, Kleczkowska HE, Strohm M and Althaus FR: Poly(ADP-ribose) binds to specific domains in DNA damage checkpoint proteins. J Biol Chem 275: 40974-40980, 2000.

39. Nie J, Sakamoto S, Song D, Qu Z, Ota K and Taniguchi T: Interaction of Oct-1 and automodification domain of poly(ADPribose) synthetase. FEBS Lett 424: 27-32, 1998.

40. Ha HC: Defective transcription factor activation for proinflammatory gene expression in poly(ADP-ribose) polymerase 1-deficient glia. Proc Natl Acad Sci USA 101: 5087-5092, 2004.

41. Villano CM and White LA: Expression of the helix-loop-helix protein inhibitor of DNA binding-1 (ID-1) is activated by alltrans retinoic acid in normal human keratinocytes. Toxicol Appl Pharmacol 214: 219-229, 2006.

42. Ling M-T, Kwok W-K, Fung MK, Xianghong W and Wong Y-C: Proteasome mediated degradation of Id-1 is associated with $\mathrm{TNF} \alpha$-induced apoptosis in prostate cancer cells. Carcinogenesis 27: 205-215, 2006.

43. Nemetski SM and Gardner LB: Hypoxic regulation of Id-1 and activation of the unfolded protein response are aberrant in neuroblastoma. J Biol Chem 282: 240-248, 2007.

44. Ogino H, Nozaki T, Gunji A, et al: Loss of Parp- 1 affects gene expression profile in a genome-wide manner in ES cells and liver cells. BMC Genomics 8: 41, 2007.

45. Carrillo A, Monreal Y, Ramírez P, Marin L, Parrilla P, Oliver FJ and Yélamos J: Transcription regulation of TNF-alpha-early response genes by poly(ADP-ribose) polymerase-1 in murine heart endothelial cells. Nucleic Acids Res 32: 757-766, 2004. 